\title{
Differential restoration of functional hyperemia by antihypertensive drug classes in hypertension-related cerebral small vessel disease
}

\author{
Masayo Koide,, ${ }^{1,2}$ Osama F. Harraz,, ${ }^{1,2}$ Fabrice Dabertrand, ${ }^{1,3,4}$ Thomas A. Longden, ${ }^{1,5}$ Hannah R. Ferris, ${ }^{1}$ Ceorge C. Wellman, ${ }^{1}$ \\ David C. Hill-Eubanks, ${ }^{1}$ Adam S. Greenstein, ${ }^{1,2,6,7}$ and Mark T. Nelson ${ }^{1,2,6,7}$ \\ 'Department of Pharmacology, Larner College of Medicine, and ${ }^{2}$ Vermont Center for Cardiovascular and Brain Health, University of Vermont, Burlington, Vermont, USA. ${ }^{3}$ Department of Anesthesiology and \\ ${ }^{4}$ Department of Pharmacology, University of Colorado Anschutz Medical Campus, Aurora, Colorado, USA. ${ }^{5}$ Department of Physiology, University of Maryland School of Medicine, Baltimore, Maryland, USA. \\ ${ }^{6}$ Division of Cardiovascular Sciences, School of Medical Sciences and ${ }^{7}$ Geoffrey Jefferson Brain Research Centre, The Manchester Academic Health Science Centre, Northern Care Alliance NHS Group, University \\ of Manchester, Manchester, United Kingdom.
}

Dementia resulting from small vessel diseases (SVDs) of the brain is an emerging epidemic for which there is no treatment. Hypertension is the major risk factor for SVDs, but how hypertension damages the brain microcirculation is unclear. Here, we show that chronic hypertension in a mouse model progressively disrupts on-demand delivery of blood to metabolically active areas of the brain (functional hyperemia) through diminished activity of the capillary endothelial cell inward-rectifier potassium channel, Kir2.1. Despite similar efficacy in reducing blood pressure, amlodipine, a voltage-dependent calciumchannel blocker, prevented hypertension-related damage to functional hyperemia whereas losartan, an angiotensin II type 1 receptor blocker, did not. We attribute this drug class effect to losartan-induced aldosterone breakthrough, a phenomenon triggered by pharmacological interruption of the renin-angiotensin pathway leading to elevated plasma aldosterone levels. This hypothesis is supported by the finding that combining losartan with the aldosterone receptor antagonist eplerenone prevented the hypertension-related decline in functional hyperemia. Collectively, these data suggest Kir2.1 as a possible therapeutic target in vascular dementia and indicate that concurrent mineralocorticoid aldosterone receptor blockade may aid in protecting against late-life cognitive decline in hypertensive patients treated with angiotensin II type 1 receptor blockers.

\section{Introduction}

Small vessel diseases (SVDs) of the brain are among the most common causes of memory loss, implicated in more than $40 \%$ of dementia cases (1-3). The principal risk factor for the development of SVDs is hypertension (4), with numerous clinical studies indicating that elevated mid-life blood pressure (BP) is associated with late-life cognitive decline $(2,5-8)$. However, the cellular mechanisms linking hypertension to memory disturbance are not yet definitively established; consequently, treatments specifically aimed at alleviating SVDs do not exist. The SPRINT-MIND study, a clinical trial involving over 9,000 hypertensive patients, showed that intensively lowering BP reduces the incidence of mild cognitive impairment (9), the forerunner to dementia. Although this study demonstrated that hypertension is a modifiable risk factor, it did not consider the varying degrees of cerebrovascular protection offered by different antihypertensive drug classes $(10,11)$. Importantly, once dementia is established, lowering BP does not appear to improve cognition $(12,13)$.

Related Commentary: https://doi.org/10.1172/JCI153202

Conflict of interest: The authors have declared that no conflict of interest exists. Copyright: (5) 2021, American Society for Clinical Investigation.

Submitted: February 24, 2021; Accepted: July 28, 2021; Published: September 15, 2021. Reference information: / Clin Invest. 2021;131(18):e149029.

https://doi.org/10.1172/JCl149029.
Maintaining healthy brain function requires precise control of cerebral blood flow (CBF) so as to support delivery of oxygen and nutrients to regions of high neuronal metabolic demand (14). This activity-dependent increase in local tissue perfusion (functional hyperemia) is supported by mechanisms collectively termed neurovascular coupling (NVC) (14-16). We recently demonstrated that capillaries, the smallest, most abundant vessels in the brain, play a pivotal role in functional hyperemia (17). Specifically, we showed that extracellular potassium $\left(\mathrm{K}^{+}\right)$, a byproduct of neural activity, activates Kir2.1 channels in capillary endothelial cells (ECs) to initiate a hyperpolarizing signal that rapidly propagates upstream, causing dilation of feeder arterioles and increasing blood flow to the region of neuronal activity.

Here, we evaluated the therapeutic effects of antihypertensive treatment with the voltage-dependent $\mathrm{Ca}^{2+}$-channel blocker amlodipine or the angiotensin II receptor type 1 (AGTR1) blocker losartan two of the most commonly used antihypertensive drugs - on hypertension-induced deficits in functional hyperemia, focusing specifically on the capillary-to-arteriole vasodilatory signaling mechanism. To this end, we used BPH mice, a polygenic model of hypertension (18) that also displays SVD (19) and cognitive impairment (20), and their corresponding normotensive control BPN mice.

\section{Results and Discussion}

At all ages examined, mean arterial pressure was approximately $25 \%$ higher in $\mathrm{BPH}$ mice than in BPN mice (Figure 1, A and B, 
A

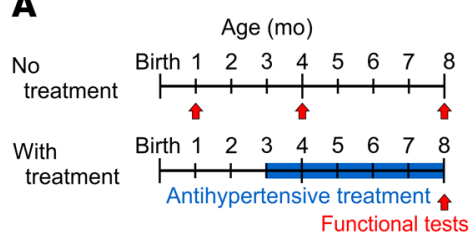

D

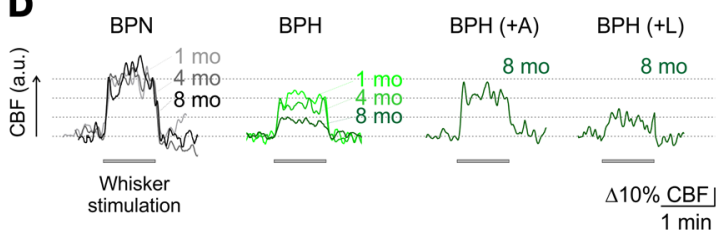

$\mathbf{F}$

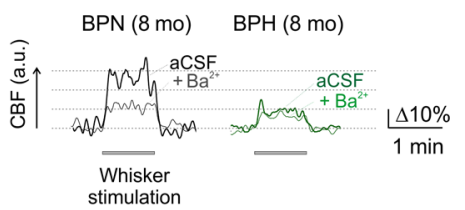

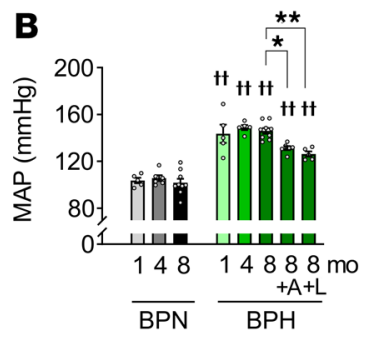
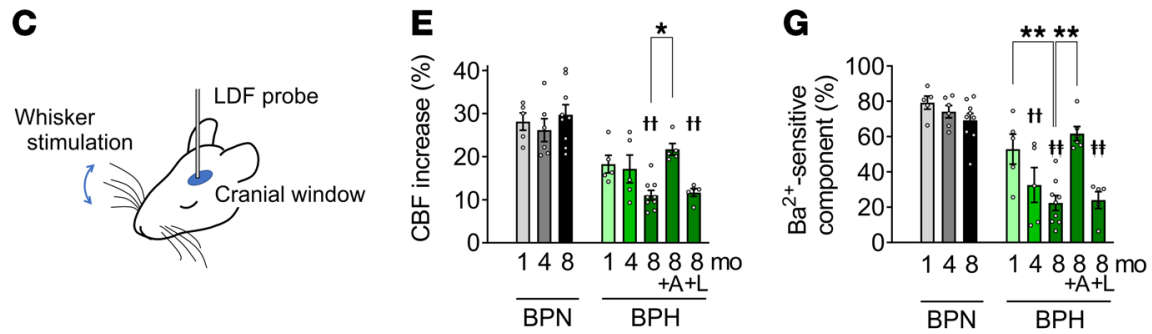

Figure 1. Hypertension-related decline in functional hyperemia is prevented by amlodipine but not losartan. (A) Experimental timelines. (B) Mean arterial pressure (MAP) in anesthetized male BPN and BPH mice at 1, 4, and 8 months of age, with and without treatment with amlodipine (BPH+A, 10 $\mathrm{mg} / \mathrm{kg} / \mathrm{d}$ ) or losartan $(\mathrm{BPH}+\mathrm{L}, \sim 100 \mathrm{mg} / \mathrm{kg} / \mathrm{d})$ starting at 3 months of age. Data are presented as mean $\pm \mathrm{SEM}\left(n=5-9\right.$ mice/group). ${ }^{+\dagger} P<0.01 \mathrm{vs}$. BPN at corresponding ages; ${ }^{*} P<0.05,{ }^{*} P<0.01$ between groups; 1 -way ANOVA followed by Tukey's test. (C) Experimental scheme for measurement of whisker stimulation-induced functional hyperemia using laser-Doppler flowmetry (LDF). (D) Functional hyperemic responses, shown as CBF increases during whisker stimulation, in male BPN and BPH mice at 1, 4, and 8 months old, and 8-month-old BPH mice with antihypertensive treatment. (E) Age-dependent progression of functional hyperemia deficits in male BPH mice, with and without antihypertensive treatment. Data are presented as mean \pm SEM ( $n=5$-9 mice/group). ${ }^{\dagger+} P<0.01$ vs. BPN at corresponding ages; ${ }^{*} P<0.05$ between groups; 1 -way ANOVA followed by Tukey's test. (F) Representative traces showing whisker stimulation-induced functional hyperemia before and after cortical superfusion of Ba ${ }^{2+}$. aCSF, artificial cerebrospinal fluid. (G) Summary data showing the $\mathrm{Ba}^{2+}$-sensitive component of whisker stimulation-induced functional hyperemia in male BPN and BPH mice, with and without antihypertensive treatment. Data are presented as mean \pm SEM ( $n=5-9$ mice/group). ${ }^{\dagger \dagger} P<0.01$ vs. BPN at corresponding ages; ${ }^{*} P<0.01$ between groups; 1-way ANOVA followed by Tukey's test.

and Supplemental Table 1; supplemental material available online with this article; https://doi.org/10.1172/JCI149029DS1). Treatment with amlodipine or losartan starting at 3 months of age substantially lowered BP in BPH mice (Figure 1, A and B, and Supplemental Table 1).

Functional hyperemia was assessed by measuring whisker stimulation-induced changes in $\mathrm{CBF}$ using a cranial window model (Figure 1C). While preserved in BPN mice, functional hyperemia progressively declined equivalently with age in both male and female BPH mice (Figure 1, D and E, Supplemental Figure 1, and Supplemental Tables 2-6), confirming hypertension-induced disruption of NVC. Subsequent studies focused on male mice. Treatment with amlodipine, but not losartan, prevented deterioration of functional hyperemia in BPH mice, despite broadly equivalent effects of both drugs on BP (Figure 1, $\mathrm{D}$ and $\mathrm{E}$ ). To determine whether the impaired functional hyperemia in $\mathrm{BPH}$ mice reflected disruption of capillary-to-arteriole signaling, we cortically superfused barium $\left(\mathrm{Ba}^{2+}\right)$, a Kir2.1 channel pore blocker (17). Application of $\mathrm{Ba}^{2+}$ blocked approximately $80 \%$ of the functional hyperemic response in normotensive BPN mice at all ages (Figure 1, F and G), in keeping with the key role of the Kir2.1 channel in functional hyperemia. In contrast, the $\mathrm{Ba}^{2+}$-sensitive component of this response was significantly educed in $\mathrm{BPH}$ mice (Figure $1, \mathrm{~F}$ and $\mathrm{G}$ ), suggesting that the Kir2.1-mediated signaling pathway is the target of hypertension-induced functional hyperemia deficits. Strikingly, chronic amlodipine treatment restored the $\mathrm{Ba}^{2+}$-sensitive component of functional hyperemic responses in $\mathrm{BPH}$ mice, whereas losartan treatment did not (Figure 1, D-G).
To further study the mechanisms underlying these observations, we used a previously published capillary-to-parenchymal arteriole (CaPA) preparation (17), an ex vivo approach in which a brain parenchymal arteriole from the middle cerebral artery territory is dissected from the brain with intact capillaries attached, mounted on a glass cannula, and pressurized to physiological pressure. The resulting pressure-induced $(40 \mathrm{mmHg})$ constriction (myogenic response) mimics the tone of these arterioles in vivo. To evaluate Kir2.1 channel function within the capillary-arteriole continuum and mimic $\mathrm{K}^{+}$release during neuronal activity, we focally applied a $10 \mathrm{mM} \mathrm{K}{ }^{+}$solution onto the capillary extremities using a pressure-ejection system while recording upstream arteriolar diameter (Figure 2A). In BPN mice of all ages examined, this maneuver resulted in rapid, robust dilation of upstream arterioles (Figure 2, B and $\mathrm{C}$ ), recapitulating the capillary-to-arteriole vasodilatory mechanism that underpins functional hyperemia (17). In contrast, this response to capillary-applied $\mathrm{K}^{+}$was progressively attenuated with age in BPH mice (Figure 2, B and C). Treatment with amlodipine, but not losartan, prevented the decline in EC Kir2.1-dependent capillary-to-arteriole signaling (Figure 2, B and C).

In addition to capillary-to-arteriole signaling, the CaPA preparation allows the study of myogenic responses and dilatory responses to arteriolar stimulation with $\mathrm{K}^{+}$. This latter approach can be used to estimate the functionality of Kir2.1 channels in the arteriole, distinct from that of Kir2.1 channels in the attached capillaries (Figure 2D). Application of $\mathrm{K}^{+}$directly onto the arteriolar segment elicited dilation in vessels from BPN mice, but not in vessels from $\mathrm{BPH}$ mice, regardless of antihypertensive treatment (Figure 2, E and F). Parenchymal arteries from $\mathrm{BPH}$ mice also 
A

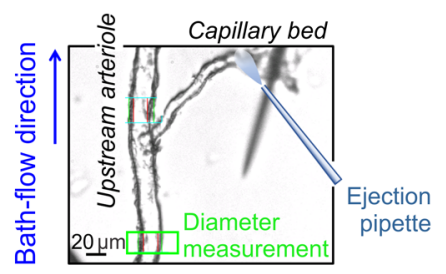

D

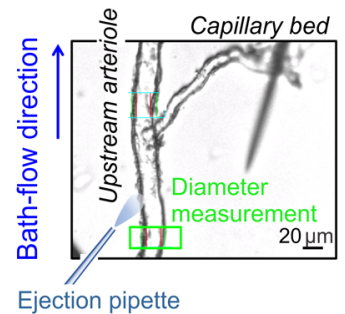

B

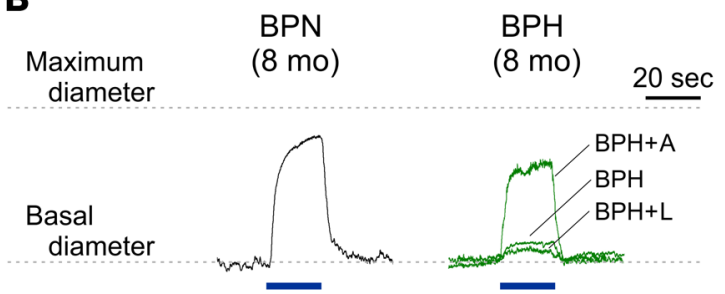

$10 \mathrm{mM} \mathrm{K}^{+}$stimulation onto capillary bed

E

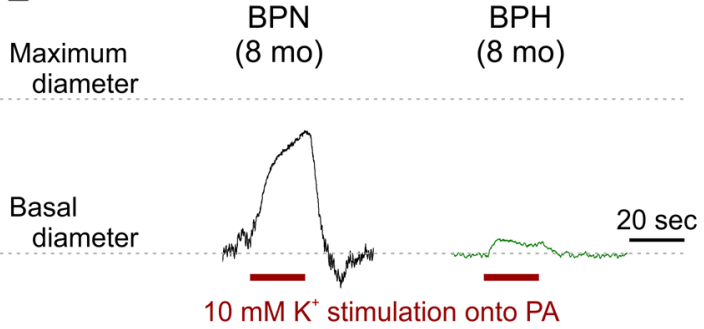

C

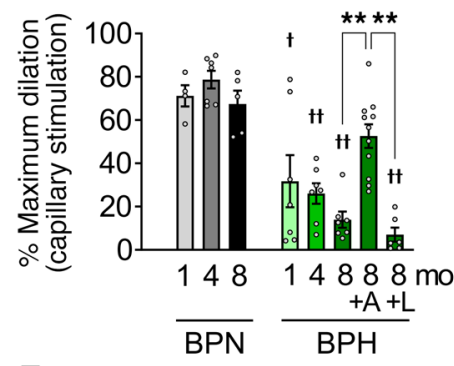

$\mathbf{F}$

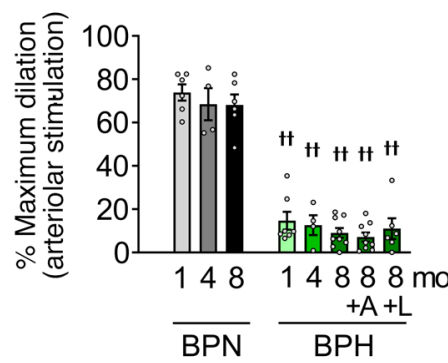

Figure 2. Capillary-to-arteriolar vasodilatory signaling is crippled in hypertension. (A) Experimental scheme showing focal stimulation of a capillary in the ex vivo CaPA preparation by pressure ejection of a $10 \mathrm{mM} \mathrm{K}{ }^{+}$artificial cerebrospinal fluid solution via an approximately 5 - $\mu \mathrm{m}$-tip glass micropipette and subsequent recording of dilation of the upstream parenchymal arteriole (green box). (B) Representative traces of upstream arteriolar dilatory response to $10 \mathrm{mM} \mathrm{K}^{+}$stimulation of capillaries in CaPA preparations from 8-month-old BPN mice, BPH mice, and BPH mice treated with amlodipine (BPH+A) or losartan (BPH+L). (C) Age-dependent attenuation of arteriolar dilatory response to capillary stimulation with $10 \mathrm{mM} \mathrm{K}+$ in $\mathrm{BPH}$ mice, with and without antihypertensive treatment. Data are presented as mean \pm SEM $\left(n=4-9\right.$ mice/group). ${ }^{\dagger} P<0.05,{ }^{\dagger \dagger} P<0.01$ vs. BPN at corresponding ages; ${ }^{* *} P<0.01$ between groups; 1-way ANOVA followed by Tukey's test. (D) Experimental scheme showing arteriolar dilatory response to application of $10 \mathrm{mM} \mathrm{K} \mathrm{K}^{+}$directly onto the arteriole. (E) Representative traces of arteriolar dilation induced by application of $10 \mathrm{mM} \mathrm{K}$ onto the arteriole segment in CaPA preparations from 8-month-old BPN and BPH mice. (F) Hypertension-induced decrease in arteriolar dilatory response to direct stimulation of arterioles with $10 \mathrm{mM} \mathrm{K}$, with and without antihypertensive treatment. Data are presented as mean \pm SEM ( $n=4-9$ mice/group). ${ }^{\dagger \dagger} P<0.01$ vs. BPN at corresponding ages by 1 -way ANOVA followed by Tukey's test.

exhibited greater myogenic tone compared with those from BPN mice, and this enhanced constriction persisted despite treatment with amlodipine or losartan (Supplemental Figure 2). The fact that amlodipine prevented the deterioration in functional hyperemia in BPH mice despite the persistence of enhanced myogenic tone and diminished arteriolar dilation to directly applied $\mathrm{K}^{+}$emphasizes the primacy of the capillary Kir2.1 channel and the capillary-to-arteriole vasodilatory mechanism in the maintenance of NVC. Consistent with this view, whereas losartan and amlodipine similarly reduced BP to an extent sufficient to prevent the development of cerebrovascular disease in a clinical setting, losartan was unable to restore capillary-to-arteriole vasodilatory signaling, and NVC remained compromised.

In agreement with in vivo and ex vivo observations of impaired capillary Kir2.1-dependent signaling, Kir2.1 currents, measured by patch-clamp electrophysiology, were significantly reduced in freshly isolated capillary ECs from BPH mice compared with those from BPN mice (Figure 3, A-C). Again, treatment with amlodipine restored Kir2.1 currents in capillary ECs, whereas losartan treatment did not (Figure 3C).

The differential impact of losartan and amlodipine on functional hyperemia closely aligns with clinical observations. Meta-analyses of drug trials in hypertensive patients have shown that calcium-channel blockers provide greater cerebrovascular protection than angiotensin-converting enzyme (ACE) inhibitors or AGTR1 blockers, particularly in the prevention of stroke (10) and dementia (11). These benefits persist over and above efficacy in reducing BP $(10,11)$. Perhaps related to this, chronic treatment with ACE inhibitors and/or AGTR1 blockers such as losartan leads to a marked elevation of aldosterone. This phenomenon, termed aldosterone breakthrough, reflects pharmacological interruption of the renin-angiotensin-aldosterone system and occurs in up to $50 \%$ of patients treated with these classes of antihypertensives (21). Accordingly, we hypothesized that the differential restoration of functional hyperemia by amlodipine and losartan in $\mathrm{BPH}$ mice - despite equivalent reductions in $\mathrm{BP}$ - may be related to aldosterone levels. To test this, we measured plasma aldosterone in 8-month-old BPN and BPH mice, with and without amlodipine or losartan treatment. We found that aldosterone levels were not significantly different in BPH mice compared with BPN mice (Figure 3D), and amlodipine treatment did not affect aldosterone levels in BPH mice (Figure 3D). In contrast, losartan treatment induced a robust increase in plasma aldosterone levels in $\mathrm{BPH}$ mice (Figure 3, D and E), consistent with clinical studies. To further investigate the effect of aldosterone breakthrough on NVC, we studied mice treated with losartan and the mineralocorticoid receptor antagonist eplerenone (Supplemental Table 7). Notably, the addition of eplerenone prevented the decline in functional hyperemia observed in mice treated with losartan alone (Figure 3, F and G), an effect that appeared to be attributable to preservation of the $\mathrm{Ba}^{2+}$-sensitive component, consistent with improvement in Kir2.1 function (Figure 3H). 
A
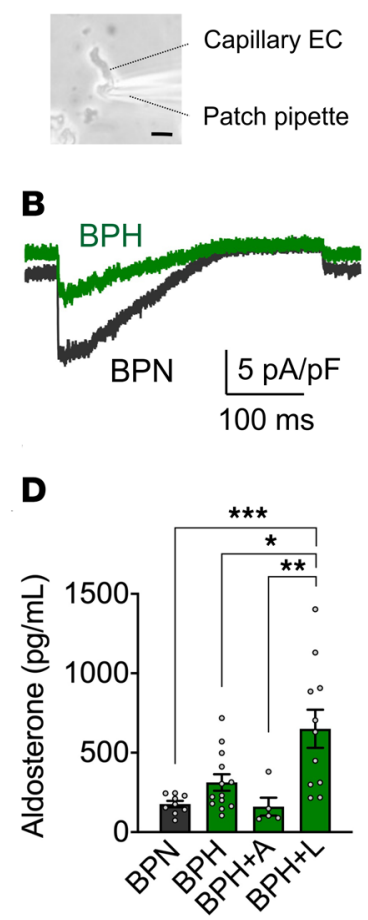

$\mathbf{F}$

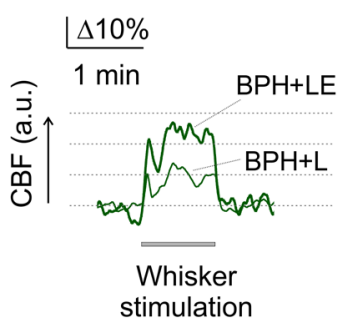

C

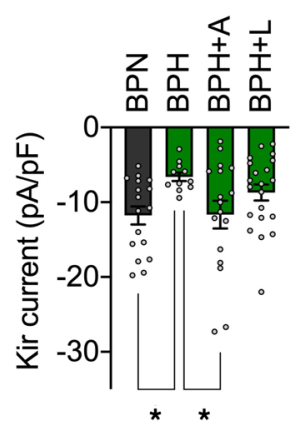

E

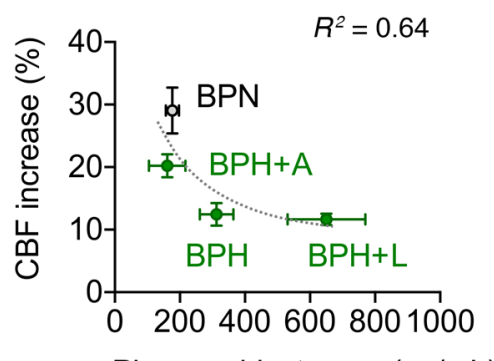

Plasma aldosterone $(\mathrm{pg} / \mathrm{mL})$

G

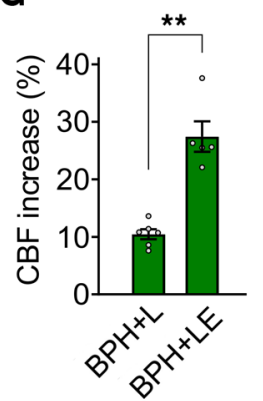

H

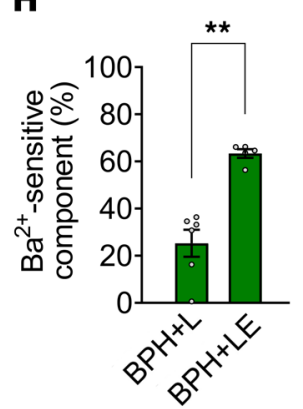

Figure 3. Capillary EC Kir2.1 channel downregulation underlies deficits in capillary-to-arteriolar signaling, which is damaged by aldosterone. (A) Patch-clamp electrophysiology in freshly isolated capillary ECs. Scale bar: $10 \mu \mathrm{m}$. (B) Representative traces of Kir2.1 currents in capillary ECs from 8-month-old BPN (black) and BPH (green) mice. (C) Summary data showing inward Kir2.1 currents (at -140 mV) in capillary ECs from 8-month-old BPN mice, BPH mice, and BPH mice treated with antihypertensives. Data are presented as mean \pm SEM ( $n=11-21$ cells/group). ${ }^{*} P<0.05$ between groups by 1-way ANOVA followed by Dunnett's multiple comparisons test. (D) Plasma aldosterone concentrations in 8-month-old mice. Data are presented as mean \pm SEM $(n=$ 5-13 mice/group). ${ }^{*} P<0.05,{ }^{* *} P<0.01,{ }^{* *} P<0.001$ between groups by 1 -way ANOVA followed by Tukey's test. (E) Correlation between plasma aldosterone levels and whisker stimulation-induced increases in local CBF ( $n=5-13$ mice/group). (F) Representative traces showing whisker stimulation-induced $C B F$ responses in mice treated with losartan $(\mathrm{BPH}+\mathrm{L})$ compared with those in mice treated with losartan and eplerenone (BPH+LE). ( $\mathbf{G}$ and $\mathbf{H})$ Restoration of total $(\mathbf{C})$ and $\mathrm{Ba}^{2+}$-sensitive $(\mathbf{H})$ functional hyperemia in $\mathrm{BPH}$ mice by combined treatment with losartan and eplerenone $(\mathrm{BPH}+\mathrm{LE})$, but not by treatment with losartan alone $(\mathrm{BPH}+\mathrm{L})$. Data are presented as mean \pm SEM ( $n=5-6$ mice/group). ${ }^{* *} P<0.01$ between groups by unpaired $t$ test.

Although amlodipine or losartan plus eplerenone treatment of $\mathrm{BPH}$ mice prevented the progressive deterioration of functional hyperemia seen in untreated BPH mice, it did not restore functional hyperemia to normotensive BPN levels. One possible explanation for this may be related to the study design, in which anti- hypertensive drugs were administered from 3 months of age. Clinical studies have highlighted the long-term adverse effects of childhood and adolescent hypertension on the cardiovascular system (22), even suggesting an association between lifetime systolic BP burden and cognitive function in middle age (23). As such, this 2- to 3-month period of elevated BP may have been sufficient to irreversibly compromise NVC for the lifetime of BPH mice, even at this relatively young mouse age (24). Future studies could consider commencing antihypertensive therapies at an earlier age. Alternatively, whereas amlodipine restored Kir2.1 currents in the capillary endothelium, additional pathological processes contribute to the overall neurovascular uncoupling seen in hypertension, such as the generation of reactive oxygen species by activated macrophages (19). This dual-hit hypothesis intimates that future treatment to prevent cognitive decline in patients with hypertension could be multifaceted, employing agents such as amlodipine in tandem with immunomodulatory therapies targeting perivascular macrophages to optimize the recovery of functional hyperemia.

It is not currently clear how elevated aldosterone levels contribute to the failure of losartan to prevent damage to capillary Kir2.1-driven functional hyperemia. However, aldosterone, a steroid hormone that contributes to BP regulation via mineralocorticoid receptors, is known to exert pleiotropic effects across the vasculature, triggering profibrotic changes and microvascular dysfunction (25). We therefore postulate that hyperaldosteronemia directly or indirectly prevents protection of the EC Kir2.1-dependent capillary-to-arteriole signaling pathway associated with BP lowering and may account for the failure of losartan to restore functional hyperemia in BPH mice. From a broader clinical perspective, losartan-induced hyperaldosteronemia and the subsequent failure of losartan to restore capillary-to-arteriole signaling could account for the excess cerebrovascular morbidity observed in patients treated with AGTR1 blockers relative to those treated with amlodipine (10, 11). It will also be important to determine whether prevention of damage to functional hyperemia in $\mathrm{BPH}$ mice derives from a direct effect of amlodipine on Kir2.1 channel function or whether the improvement in function is merely due to amlodipine's BP-lowering effect. However, improvements in functional outcomes in mice treated with losartan plus eplerenone suggest the latter of these possibilities, namely, that it is the level of BP that triggers the damage to capillary endothelial Kir2.1mediated NVC. One possible target for both hypertension and aldosterone is the phospholipid $\mathrm{PIP}_{2}$, which is an essential cofactor for Kir2.1 channels (26). In support of this, deficits in functional hyperemia in mouse models of Alzheimer's and SVDs are caused by $\mathrm{PIP}_{2}$-mediated loss of Kir2.1 electrical signaling in capillary ECs $(27,28)$.

A final important consideration, particularly with regard to the translational applicability of our findings, is that the BPH mouse 
is a polygenic and idiopathic model of hypertension that does not display salt sensitivity (29), attributes that are common to approximately $50 \%$ of patients with hypertension (30). However, it will be important to validate crippling of the capillary endothelial Kir2.1 channel-mediated NVC mechanism in alternative animal models of hypertension and confirm the divergent restorative properties of antihypertensive drug classes.

In summary, we show that chronic hypertension in $\mathrm{BPH}$ mice causes neurovascular uncoupling - a hallmark of SVDs of the brain - through downregulation of brain capillary EC Kir2.1 channel activity (Supplemental Figure 3). By suppressing the function of this channel, chronic hypertension disables a key mechanism by which the brain microcirculation detects local neuronal activity and increases local blood flow to metabolically active regions. Consistent with human studies, reducing systemic BP alone was not sufficient to prevent hypertension-related defects in capillary-to-arteriolar signaling. Specifically, whereas losartan effectively lowered $\mathrm{BP}$ in $\mathrm{BPH}$ mice, it failed to prevent damage to the capillary-to-arteriole signaling mechanism at the heart of NVC, likely due to hyperaldosteronism. In contrast, treatment with amlodipine, which is more effective than losartan in preventing some types of cerebrovascular diseases, reduced $\mathrm{BP}$ and prevented progressive hypertension-related damage to capillary Kir2.1-dependent NVC. From a clinical perspective, these data suggest the need for new drug trials that exploit the greater efficacy of amlodipine relative to losartan in preventing vascular dementia in hypertensive patients. Alternatively, the addition of mineralocorticoid receptor antagonists could be studied in patients already on ACE inhibitors or AGTR1 blockers, with the express purpose of blocking the effects of elevated aldosterone and protecting the brain.

\section{Methods}

Animal studies. Hypertensive BPH and normotensive BPN mice were used throughout. Functional hyperemia was examined by laserDoppler flowmetry; capillary-arteriole functionality was evaluated by ex vivo arteriography; and Kir channel currents were measured electrophysiologically using the conventional whole-cell configuration of the patch-clamp technique. Detailed information is provided in Supplemental Methods.

Statistics. Data are presented as mean \pm SEM. For multiple comparisons, statistical analyses were accomplished using 1-way analysis of variance (ANOVA) followed by Tukey's post hoc multiple comparisons test, unless otherwise stated. Unpaired Student's $t$ test was used for 2 -group analyses. $P$ values of less than 0.05 were considered statistically significant.

Study approval. All experimental protocols used in this study complied with ARRIVE guidelines and were approved by the Institutional Animal Care and Use Committee of the University of Vermont.

\section{Author contributions}

MK designed and directed the research, performed in vivo and ex vivo experiments and aldosterone ELISAs, and prepared the manuscript. FD established the ex vivo preparation and acquired ex vivo data. OFH and TAL acquired and analyzed electrophysiology data. HRF performed tail-cuff BP measurements. GCW and DCHE edited the manuscript. ASG contributed to research design and manuscript preparation. MTN designed and directed the research and prepared the manuscript. All authors approved its submission.

\section{Acknowledgments}

This study was supported by the American Heart Association (14SDG20150027 to MK; 17SDG33670237 to TAL, 17POST33650030 and 20CDA35310097 to OFH); the Million Dollar Bike Ride Pilot Grant Program (MDBR-21-101-CADASIL to MK); the Totman Medical Research Trust (to GCW and MTN); the Fondation Leducq (Pathogenesis of Small Vessel Disease of the Brain to MTN); the European Union Horizon 2020 Research and Innovation Program SVDs@target (no. 666881 to MTN); the British Heart Foundation (PG/18/7/33535, FS/19/8/34163 to ASG); the Fred and Maureen Done Charitable Trust (to ASG); and the NIH (P2O-GM-135007 to MK, OFH and MTN; R01-HL-136636 to FD; R01-AG066645 and DP2NS121347 to TAL; R01-HL-142888 to GCW; P01-HL-095488 to GCW and MTN; R35-HL-140027, R01NS-110656, R01-HL-121706, R37-DK-053832,7UM-HL-1207704, and R01-HL-131181 to MTN).

Address correspondence to: Mark T. Nelson, Given Building, Room B-333A, 89 Beaumont Avenue, Burlington Vermont 054050068, USA. Phone: 802.656.2500; Email: Mark.Nelson@uvm. edu. Or to: Masayo Koide, Given Building, Room B-322A, 89 Beaumont Avenue, Burlington Vermont 05405-0068, USA. Phone: 802.656.1497; Email: Masayo.Koide@uvm.edu.
1. Snyder HM, et al. Vascular contributions to cognitive impairment and dementia including Alzheimer's disease. Alzheimers Dement. 2015;11(6):710-717.

2. Iadecola C, et al. Vascular cognitive impairment and dementia: JACC Scientific Expert Panel. JAm Coll Cardiol. 2019;73(25):3326-3344.

3. Pantoni L. Cerebral small vessel disease: from pathogenesis and clinical characteristics to therapeutic challenges. Lancet Neurol. 2010;9(7):689-701.

4. Joutel A. Prospects for diminishing the impact of nonamyloid small-vessel diseases of the brain. Annu Rev Pharmacol Toxicol. 2020;60:437-456.

5. Iadecola C, Gottesman RF. Neurovascular and cognitive dysfunction in hypertension. Circ Res.
2019;124(7):1025-1044.

6. Iadecola C, et al. Impact of hypertension on cognitive function: a scientific statement from the American Heart Association. Hypertension. 2016;68(6):e67-e94.

7. Tadic M, et al. Hypertension and cognitive dysfunction in elderly: blood pressure management for this global burden. BMC Cardiovasc Disord. 2016;16(1):208.

8. Walker KA, et al. Defining the relationship between hypertension, cognitive decline, and dementia: a review. Curr Hypertens Rep. 2017;19(3):24.

9. Group SMIftSR, et al. Effect of intensive vs standard blood pressure control on probable dementia: a randomized clinical trial. JAMA.
2019;321(6):553-561

10. Webb AJ, et al. Effects of antihypertensive-drug class on interindividual variation in blood pressure and risk of stroke: a systematic review and meta-analysis. Lancet. 2010;375(9718):906-915.

11. Staessen JA, et al. Placebo-controlled trials of blood pressure-lowering therapies for primary prevention of dementia. Hypertension. 2011;57(2):e6-e7.

12. Beishon LC, et al. The evidence for treating hypertension in older people with dementia: a systematic review. JHum Hypertens. 2014;28(5):283-287.

13. van der Wardt V, et al. Antihypertensive treatment in people with dementia. JAm Med Dir Assoc. 2014;15(9):620-629.

14. Iadecola C. The neurovascular unit coming of 
age: a journey through neurovascular coupling in health and disease. Neuron. 2017;96(1):17-42.

15. Attwell D, et al. Glial and neuronal control of brain blood flow. Nature. 2010;468(7321):232-243.

16. Nippert AR, et al. Mechanisms mediating functional hyperemia in the brain. Neuroscientist. 2018;24(1):73-83.

17. Longden TA, et al. Capillary $\mathrm{K}^{+}$-sensing initiates retrograde hyperpolarization to increase local cerebral blood flow. Nat Neurosci. 2017;20(5):717-726.

18. Jackson KL, et al. Mechanisms responsible for genetic hypertension in Schlager $\mathrm{BPH} / 2$ mice. Front Physiol. 2019;10:1311.

19. Faraco G, et al. Perivascular macrophages mediate the neurovascular and cognitive dysfunction associated with hypertension. JClin Invest. 2016;126(12):4674-4689.
20. Hartman RE, et al. Motor and cognitive deficits in mice bred to have low or high blood pressure. Physiol Behav. 2012;105(4):1092-1097.

21. Bomback AS, Klemmer PJ. The incidence and implications of aldosterone breakthrough. Nat Clin Pract Nephrol. 2007;3(9):486-492.

22. Bao W, et al. Essential hypertension predicted by tracking of elevated blood pressure from childhood to adulthood: the Bogalusa Heart Study. Am J Hypertens. 1995;8(7):657-665.

23. Razavi AC, et al. Left ventricular mass index is associated with cognitive function in middle-age: Bogalusa Heart Study. Circ Cardiovasc Imaging. 2020;13(8):e010335.

24. Ojeda NB, et al. Role of fetal programming in the development of hypertension. Future Cardiol. 2008;4(2):163-174.

25 . Gorini S, et al. Role of aldosterone and mineralo- corticoid receptor in cardiovascular aging. Front Endocrinol (Lausanne). 2019;10:584.

26. Hansen SB, et al. Structural basis of $\mathrm{PIP}_{2}$ activation of the classical inward rectifier $\mathrm{K}^{+}$channel Kir2.2. Nature. 2011;477(7365):495-498.

27. Dabertrand F, et al. $\mathrm{PIP}_{2}$ corrects cerebral blood flow deficits in small vessel disease by rescuing capillary Kir2.1 activity. Proc Natl Acad Sci U S A. 2021;118(17):e2025998118.

28. Mughal A, et al. PIP 2 improves cerebral blood flow in a mouse model of Alzheimer's disease. Function (Oxf). 2021;2(2):zqab010.

29. Leckie BJ. The action of salt and captopril on blood pressure in mice with genetic hypertension. J Hypertens. 2001;19(9):1607-1613.

30. Weinberger MH, et al. Definitions and characteristics of sodium sensitivity and blood pressure resistance. Hypertension. 1986;8(6 pt 2):II127-II134. 